\title{
Algunas reflexiones en torno a la Televisión Digital
}

\section{Terrestre}

\section{Carmen Caffarel Serra}

Catedrática de Comunicación Audiovisual y Publicidad. Departamento de Ciencias de la

Comunicación I. Facultad de Ciencias de la Información. Universidad Rey Juan Carlos.

Carmen.caffarel@urjc.es

\section{Resumen}

La televisión digital permite mejorar la calidad de la imagen, ampliar el número de canales, tener interacción con los medios de comunicación con usos parecidos a los que hoy brinda Internet y tener televisión para dispositivos móviles como teléfonos celulares, pero sobre todo, lo que se destaca en este artículo, es la disminución de la brecha digital que promueve la Televisión Digital Terrestre por su fácil accesibilidad y por la adopción, en España, de un modelo de Televisión digital Terrestre en abierto y gratuito. 


\title{
Palabras clave
}

Televisión Digital Terrestre - Sociedad de la Información - Globalización - Brecha digital -

\author{
Interactividad
}

\begin{abstract}
Digital Television improves image quality, increases the number of television channels and allows the user to access tools similar to those provided by the Internet and Television with cellar attachments. As we see, the most important aspect of Digital television is that it minimizes the digital divide, allowing its easy accessibility. These advantages have been shown in the open and free model of this digital terrestrial television that is present in Spain.
\end{abstract}

\section{Key words}

Digital Terrestrial Television - Information Society - Globalization - Digital Divide - Interactivity

\section{La Televisión Digital Terrestre: una realidad social}

La Televisión Digital Terrestre está ya en la calle. En estos días observamos multitud de anuncios institucionales en televisión, radio y prensa sobre las bondades de la televisión digital, podemos afirmar que la Televisión Digital Terrestre es de interés general y lo será más conforme se acerque la fecha del apagado analógico, en España previsto para 2010 y en Europa 2012 como fecha máxima, y 
sobre el que los aspectos tecnológicos y económicos (modelos de negocio) han sido, a nuestro juicio, mucho más debatidos hasta la fecha que sus consecuencias sociales.

Tenemos claro, sin embargo, que este fenómeno va a afectar a toda la población, a todos los sectores sociales desde los que ya están familiarizados con Internet y las Nuevas Tecnologías hasta los que por edad, por su formación o simplemente por una actitud vital les cuesta adaptarse más a los cambios sociales y tecnológicos.

Por eso decimos que la Televisión Digital Terrestre es la Televisión Digital de todos como una evolución más (una revolución habría que decir) de la misma televisión, de la vieja televisión analógica, cuyo cincuentenario en España se ha celebrado durante todo el año pasado.

Si hay un aspecto obvio de la realidad social, política, económica y cultural de este comienzo del tercer milenio en el que, con toda probabilidad, todos coincidimos, es en el enorme peso de los medios de comunicación e información sobre la vida cotidiana. Un peso que ha tomado tal dimensión que, con frecuencia, resulta -por qué no decirlo- casi abrumador.

Hemos podido seguir a través de la televisión los debates y la noche electoral de los últimos comicios norteamericanos, como si fueran nuestros debates y nuestra noche electoral. Hemos sido testigos, casi al minuto, de la guerra de Irak. La visión en directo, desde todos los rincones del planeta, de los ataques terroristas a las Torres, fue, además de una inmensa tragedia, una experiencia mediática sin precedentes en la Historia.

Todo ello nos habla de la dimensión de los cambios de todo orden que las nuevas tecnologías de la comunicación están introduciendo en nuestras vidas y nos da testimonio de la realidad con la que 
convivimos inevitablemente. Con la que conviven, quizá de una manera infinitamente más natural que nosotros, nuestros hijos. Es más: quienes hayan nacido después del año 2000, crecerán en un contexto radicalmente nuevo.

En un contexto en el que la información y, más allá, la comunicación audiovisual, aparecerán como prolongación de su propia naturaleza, como parte consustancial de su condición de seres sociales.

Estamos inmersos en la sociedad del conocimiento. En una sociedad en la que el capital humano se ha convertido en la principal riqueza. Y en la que la información es no sólo un valor creciente sino una necesidad imprescindible para el desarrollo social de los pueblos. La economía, la cultura, la política, los servicios sociales, la realidad educativa, la sanidad, el ocio, ya no son concebibles al margen del permanente fluir de la información.

Todo esto, que ya resulta una obviedad, permite una primera reflexión. En nuestras sociedades y, de manera concreta en la sociedad española de hoy, el derecho a la información y a la libertad de información ha adquirido una relevancia radical. Es, más que nunca, consustancial al concepto de democracia y debe de ser considerado como una pieza inseparable del funcionamiento cotidiano de todos los mecanismos internos del modelo de convivencia europeo y occidental.

De aquí nuestra profunda identificación con el artículo 20 de nuestra Constitución. En él no sólo se reconocen y protegen los derechos de todos los ciudadanos "a comunicar o recibir libremente información veraz por cualquier medio de difusión”. También se establece el mandato de garantizar el pluralismo de la sociedad, así como el acceso de los grupos sociales y políticos significativos a “los medios de comunicación social dependientes del Estado o de cualquier ente público”. 
Como se sabe a la etapa nacida el pasado siglo en los años 60 y 70, caracterizada por el impulso de la llamada "contracultura" y cuya vigencia se extendió hasta mediados de los noventa, ha sucedido la etapa de la "cibercultura". No son pocos los especialistas en el estudio de las dinámicas sociales que afirman, no sin razón, que a la generación de finales del siglo que Douglas Coupland definió, en 1991, como “generación X” está sucediendo la “generación @”.

Las tecnologías de última generación, que van desde Internet al Weblog pasando por la televisión y la radio digital interactivas no sólo globalizan, como temían los sectores más apocalípticos de la cultura antiglobalización, el mercado y los flujos económicos, no sólo coadyuvan a la homogeneización del pensamiento. También globalizan contenidos culturales minoritarios, posibilitan el desarrollo de iniciativas de carácter colectivo, abren posibilidades de generar información al margen de las grandes corporaciones de la comunicación. Es decir: incorporan pluralismo, diversidad, educación.

Y, lo que ha mi juicio es más importante, incorporan nuevas pautas en la búsqueda de información. Pautas que descansan en una actitud activa ante el medio: el ciudadano busca e indaga acerca de la información que quiere, se hace preguntas, y ello es posible con la Televisión Digital Terrestre. Y pautas que generan igualdad en el acceso a la cultura: con independencia del status económico, todo ciudadano puede acceder a fondos de información y documentación.

\section{En la España democrática la Televisión ha pasado por}

\section{diversas etapas}


La televisión en España, desde la democracia, ha vivido un proceso evolutivo en el que cabe considerar 5 etapas y en el que se puede advertir la distinta función que, respecto a la sociedad, juegan la televisión pública y la televisión privada.

La primera es la que va de 1975 a 1980, en la que lo esencial es la continuidad de la televisión heredada del franquismo (1956-1975) con un solo cambio de importancia. La generalización del color, lo que tuvo algo de metáfora del proceso político que se vivió entonces. Por tanto, sólo había, sin una regulación democrática, una televisión, TVE, con 2 cadenas.

La segunda etapa se inicia, en 1980 con la aprobación del Estatuto de Radio y Televisión (desde el año pasado derogada), en el que se estableció el papel fundamental de la Radio Televisión pública estatal, adaptándola a las exigencias de pluralismo, libertad de información, descentralización y acceso de la ciudadanía que se derivaban del texto constitucional.

La tercera etapa se inició en 1983, con la aprobación de la Ley del Tercer Canal y con la aparición, en el mapa audiovisual español, de las televisiones públicas autonómicas, televisiones que siguen, para su regulación, el modelo de Televisión Española vigente hasta 2006.

La cuarta etapa constituyó el gran cambio en materia de televisión. En mayo de 1988 se aprobó la Ley de la Televisión Privada, y, dos años después, en 1990 comenzaron en abierto las emisiones de Telecinco y Antena 3. El espacio radioeléctrico dejó de estar ocupado por operadores públicos para incorporar el principio de convivencia (y de competencia) entre operadores públicos y televisiones privadas. El pluralismo se instalaba en un ámbito en el que, en España, sólo había actuado la iniciativa pública y se abría paso una etapa radicalmente nueva en la que ya no habría tarta publicitaria única, ni audiencias unívocas, ni garantía de autofinanciación vía ingresos publicitarios. 
Era la etapa de la diversidad televisiva, también de la pluralidad de la oferta tanto en contenidos como en información y, por derivación, la etapa que obligaba a la industria audiovisual española (no sólo a las televisiones existentes o a las recién nacidas) a la búsqueda de nuevos caminos, de nuevas formas de producción.

Desde el año 1990 hasta 2004, en la que comienza la etapa actual la estructura de la industria televisiva en España se basa en cinco ofertas claramente diferenciadas en el mercado:

1) las 5 cadenas de televisión generalistas de ámbito nacional (2 públicas (TVE1 y la 2) y las privadas Antena 3 y Telecinco a las que habría que sumar Canal Plus, oferta de pago, que en determinadas franjas horarias ofrecía contenidos generalistas en abierto para todo el territorio nacional

2) los 12 canales autonómicos públicos distribuidos en ocho Comunidades Autónomas diferentes y federados en el organismo denominado FORTA, institución creada para defender sus intereses comunes.

3) las emisoras locales de televisión: en el mercado español, en 2004, estaban emitiendo unas 897 emisoras locales de televisión, según el censo del Estudio General de Medios. De éstas, 139 emitían en capitales de provincia, de acuerdo con el recuento realizado por la empresa GECA.

4) las ofertas de pago que llegan a través de las plataformas digitales: En 2004 operaba en nuestro país la plataforma Canal Satélite Digital: una emisora producto de la fusión de la plataforma Canal Satélite Digital, del Grupo Prisa, y la plataforma Vía Digital, del Grupo Telefónica. 
5) y la televisión por cable que se estructuraba, fundamentalmente, en torno a tres grandes grupos de operadores: ONO, IMAGENIO, AUNA. A estos habría que añadir la antigua Agrupación de Operadores de Cable.

Dejo al margen la fallida experiencia de la Televisión Digital Terrestre en esta etapa, (Quiero TV) experiencia a la que me referiré enseguida al hablar del estado actual de la TDT en España.

La quinta etapa, algunos han denominado a esta etapa, sobre todo al curso 2005-06 de refundación del sector audiovisual, supone una verdadera revolución que se inicia con el cambio de gobierno en abril de 2004. El actual presidente del gobierno decide llevar a cabo una serie de medidas para reformar el papel de los medios de comunicación públicos y privados y reorganizar el sector.

Por lo que concierne a Radio Televisión Española se inicia un profundo proceso de renovación de contenidos encaminados a devolverle la credibilidad perdida como consecuencia de la fuerte gubernamentalización de épocas anteriores, a lograr una pluralidad, y una reforma empresarial sin precedentes.

Se trataba de regenerar democráticamente tanto su gestión como sus contenidos. De trabajar por cambiar profundamente la antigua radiotelevisión y por sentar las bases que facilitaran la transición hacia un nuevo modelo:

- Ello suponía que principios como autonomía editorial, pluralismo, independencia profesional, se hicieran norma en sus informativos y en toda su oferta de contenidos. 
- Había que lograr, también, que la transparencia, la eficacia, la contención del gasto improductivo y la eficiencia fueran una práctica consolidada en la gestión de los recursos.

- Y conseguir, en fin, la apertura de nuevas vías a la participación de oyentes y telespectadores, de articular mecanismos que garantizaran su acceso, que canalizaran sus críticas.

En paralelo a esos objetivos, se planteó el gran objetivo estratégico: la reforma eternamente aplazada del Ente público RTVE. Una reforma en profundidad que habría de plasmarse en todos los niveles: en el sistema de elección de sus órganos de gobierno y administración; en el modelo de financiación, con la apertura de vías de superación de la deuda heredada; en el de la racionalización y eficiencia de su estructura; en el de los contenidos; en el de la información; en el de la innovación tecnológica.

Esa labor ha venido a confluir, con una serie de iniciativas gubernamentales de un alcance histórico sin precedentes.

Aunque el gobierno debe de ser neutral en el ámbito de actuación de los distintos medios audiovisuales, debe de ser beligerante a la hora de establecer un marco regulador, unos principios éticos de funcionamiento y mecanismos que garanticen la libertad de expresión.

Desde el punto de vista legislativo el día 5 de junio de 2006 el Congreso de los Diputados aprobó la Ley de la radio y la televisión estatal culminado un proceso que había arrancado casi tres años antes a la vez que el Estado asumía la deuda histórica de RTVE. 
Casi un año antes, a mediados de julio de 2005, entraba en vigor la ley de medidas urgentes para el impulso de la Televisión Digital Terrestre, de Liberalización de la Televisión por Cable y de Fomento del Pluralismo.

La primera consecuencia de este nuevo marco legal fue que aparecieron en la escena televisiva española dos nuevas cadenas en abierto y gratuitas: Cuatro y la Sexta. Se rompía así el duopolio que mantenían Antena 3 y Telecinco en el sector de la televisión privada en abierto.

En efecto, el 7 de noviembre de 2005 Cuatro arranca sus emisiones en abierto con una sintonización de prácticamente el $100 \%$ al ocupar la frecuencia analógica de Canal + que queda solamente como operador de pago. El 27 de marzo de 2006, la Sexta comienza sus emisiones pero con una gran diferencia, apenas llega a los hogares españoles y tiene que iniciarse un progresivo proceso de antenización que, sin lugar a dudas, se ve impulsado al adquirir la Sexta a Telefónica, los derechos de retransmisión del mundial de fútbol de Alemania en junio-julio de 2006.

Con la aparición de estos dos nuevos operadores España se convierte en el mercado más diversificado y plural de Europa: En analógico con seis cadenas de ámbito nacional en abierto y gratuitas, dos públicas del mismo grupo (TVE 1, La 2) y cuatro privadas (Antena 3, Telecinco, Cuatro, La Sexta). A ello hay que sumar las televisiones autonómicas que se han visto incrementadas en cinco comunidades autónomas más (Asturias, Aragón, Baleares, Extremadura y Murcia) La consecuencia más obvia ha sido la fragmentación de la audiencia y la disminución de las cuotas de liderazgo de las cadenas, proceso que reproduce las tendencias experimentadas en Europa, donde el liderazgo se obtiene con cuotas de entre el 10 y el $12 \%$. 
Por lo que concierne a la Televisión Digital Terrestre la ley antes mencionada se completa con el Plan técnico de la Televisión Digital Terrestre (RD de 29 de julio de 2005) que dibuja un nuevo mapa en su implantación y permite que el día 30 de noviembre de ese mismo año los espectadores españoles puedan sintonizar 18 canales de ámbito estatal gratuitos. Vamos a detenernos en su explicación, en su breve historia y en sus ventajas.

La televisión digital terrestre representa la evolución de la televisión tal y como hoy la conocemos al formato digital. El proceso de transición de la televisión analógica a la digital terrestre ha venido inicialmente marcado por el interés de los gobiernos para aprovechar de forma más eficiente el espectro actualmente utilizado por la televisión analógica, por ampliar la oferta de canales, y por impulsar los nuevos servicios y facilidades (interactividad y en un futuro Internet) que podrá ofrecer la televisión digital. En este interés se marcó un calendario de transición que debía concluir en el denominado “apagón analógico", que en España se fijó inicialmente en el 2012 y ha sido adelantado al año 2010.

\section{La Televisión Digital terrestre en España}

El Plan Técnico de 1998, por tanto un plan de la etapa anterior, situaba a España en la vanguardia del desarrollo de la Televisión Digital Terrestre, pero apostando por un modelo de TV de pago. Se asignaron a QUIERO TV, tres y medio múltiples digitales, casi la totalidad del espectro que se había planificado a nivel nacional para el lanzamiento de la TDT. 
Veo Televisión (consorcio liderado por Recoletos y el periódico El Mundo), y Net Televisión que es una oferta sustentada por el Grupo Vocento y Pantalla Digital, entre otros, consiguieron cada uno un canal digital gratuito, completando de este modo medio múltiple que compartían con Quiero TV.

Mientras tanto los radiodifusores nacionales ya instalados en analógico (TVE, Antena 3, Telecinco y Canal +) quedaban relegados a compartir un múltiple digital, por el que obligatoriamente debían emitir sus programas generalistas en precarias condiciones técnicas de emisión, no olvidemos que estamos hablando de cinco programas puesto que TVE emitía TVE 1 y La 2,

Quiero TV llegó a disponer en su mejor momento de unos 200.000 abonados. Al cabo de dos años de funcionamiento presentó la declaración de quiebra y devolvió la licencia. A mediados de 2002 se cerraba este primer intento de lanzar en España la TDT bajo un modelo de suscripción o de pago.

Es curioso constatar un cierto paralelismo entre esta experiencia y la del Reino Unido donde, por esas mismas fechas, cerraba también sus emisiones On Digital, la marca de ITV para la TDT de pago. Sin embargo, y a diferencia del Reino Unido, donde sólo unos meses más tarde se puso en marcha la plataforma gratuita de la TDT, Freeview, en España habría que esperar más de tres años, estamos hablando de 2005, para que se retomara el lanzamiento de la TDT.

En enero de 2005 en una Conferencia pronunciada en el Forum Europa de Nueva Economía en Madrid, en mi condición de Directora General de RTVE, decía

Radio Televisión Española reclama la posición de protagonismo decidido en el relanzamiento de la Televisión Digital Terrestre. Este papel, consustancial con su definición de servicio público, no pretende excluir a otros actores. Les recuerdo que, de 
este modo, en la futura plataforma española de TDT puede haber 12 programas nacionales producidos por empresas comerciales y 8 por RTVE, a los que se unirían los programas autonómicos y locales, públicos y privados.

Podemos adelantarnos en la fase de transición y desempeñar el papel de "locomotora" que desde muchos ámbitos se nos ha demandado, dado que estamos preparados con nuestra producción actual para poder utilizar los dos múltiplex desde el principio de la reasignación de canales $(. .$.

Rotundamente quiero afirmar que RTVE sabe, puede y quiere liderar la nueva Televisión Digital Terrestre, que será la televisión del siglo XXI, una televisión abierta, gratuita, plural, con capacidad para ser un elemento de desarrollo de la Sociedad de la Información.

El segundo Plan Técnico de la TDT, el del año 2005, establece un escenario para el momento de cierre de las emisiones digitales en el que se otorga un múltiple digital completo a cada uno de los operadores nacionales de televisión que ya están presentes en el actual escenario audiovisual, salvo en el caso de RTVE, que le se asignan dos múltiples completos, uno de ellos (igual que ocurre en estos momentos) con posibilidad de realizar desconexiones de ámbito autonómico.

Se consolida así en España un modelo de Televisión digital gratuita con un mínimo de 3treinta y dos canales nacionales ${ }^{1}$ a los que hay que añadir otros ocho de ámbito autonómico y cuatro canales más de carácter local en cada una de las 281 demarcaciones municipales que se han planificado en el conjunto del país, lo que supone más de 1.100 canales locales de televisión.

\footnotetext{
${ }^{1}$ Cuatro para la Sexta, cuatro para VEO TV, cuatro para Net TV, cuatro para Antena 3, cuatro para Tele5, cuatro para Sogecable, 8 para TVE.
} 
Esto implica que cada español, en 2010, podrá ver en su domicilio, de forma gratuita, entre 44 y 52 canales distintos, entre nacionales autonómicos y locales.

Recapitulando: a diferencia de la fallida experiencia de Quiero TV, el lanzamiento de la Televisión Digital Terrestre en España ha vuelto a nacer bajo nuevas pautas:

1. Un modelo de TV gratuita

2. Un modelo basado en los radiodifusores ya existentes, aunque se permite la entrada de otros nuevos.

3. Un modelo que convierte a las cadenas de ámbito nacional, públicas y privadas en los motores del cambio, haciendo de locomotora para los canales locales y autonómicos y no al revés.

Con la finalidad de promover la transición hacia la TDT a finales de 2005 se constituyó Impulsa TDT, nombre comercial de la Asociación para la Implantación y Desarrollo de la Televisión Digital Terrestre que aglutina a los radiodifusores de ámbito nacional y autonómico, y el principal operador de la red de difusión (Abertis), y en colaboración permanente con la Administración, en concreto el 7 de junio de 2006 Impulsa TDT firmó un convenio con el Ministerio de Industria, Turismo y Comercio con el objeto de establecer un marco que permita la ejecución de actuaciones conjuntas o coordinadas para el impulso de la Televisión Digital Terrestre en España.

Los principales países europeos han optado también por la Televisión Digital Terrestre en abierto y gratuito o un sistema mixto gratuito y de pago. Valga a modo de ejemplo el modelo británico que se relanza a finales de 2002, pocos meses después del fracaso de la plataforma de pago, y lo hace bajo 2 premisas: 
- un modelo gratuito, con amplia oferta de canales

- una plataforma abierta, free, liderada por la BBC

Cuatro años después, con más de diez millones de receptores de la Televisión Digital Terrestre en los hogares y un plan de apagado ya en marcha, previsto entre los años 2008 y 2012, Freeview ha resultado un modelo de éxito que se ha convertido en referencia para otros países europeos.

Buena parte de ese éxito se debe, sin duda, al papel jugado por la BBC que, a través de dos múltiples digitales completos, emite una atractiva oferta de contenidos basada en tres pilares:

- la información (un canal de noticias de 24 horas y un canal de información interactiva a través del propio televisor)

- la atención a la infancia (dos canales infantiles: uno para niños de preescolar, hasta los cinco años, y el otro de seis a trece años)

- el entretenimiento: un canal especial para jóvenes (BBC 3) y otro para un público más maduro (BBC 4).

Junto a ello, la BBC ofrece en su múltiple el canal parlamentario, una ventana abierta a los temas sociales y al voluntariado (Community Channel), once emisiones radiofónicas de la BBC y los dos canales generalistas (BBC 1 y BBC 2) que se emiten tanto en analógico como en digital.

De forma complementaria a la plataforma gratuita, desde hace dos años existe en el Reino Unido una oferta de TDT de pago, con once canales adicionales. 
En España las emisiones, en TDT, iniciadas a finales de 2005 se han venido consolidando y al día de hoy, según los datos aportados por diversos especialistas, casi nueve millones de personas ven la televisión a través de ese nuevo cauce tecnológico. En el último informe de penetración de la TDT del plan impulsa indica que la TDT obtiene un 5,7 \% de share en marzo. Un 80,45\% de antenización cubierta; 4,1 millones de sintonizadores vendidos al mes de marzo; 17,6\% de hogares disponen de acceso a la TDT.

En cualquier caso es mayor la elección de los telespectadores por las ofertas temáticas, menos por las generalistas que, como es obvio por el nivel de implantación, tienen todavía, mayor audiencia en analógico. Veremos qué ocurre cuando se produzca el apagón analógico en 2010 y todos los contenidos generalistas y temáticos compitan en igualdad de condiciones.

Es necesario resaltar una de las características que, en nuestra opinión, van a marcar la diferencia de la TV Digital con respecto a la analógica: la interactividad.

La interactividad es un valor añadido a la programación convencional, abre la puerta a la participación en los contenidos audiovisuales y además sitúa al viejo aparato de televisión en el centro de la revolución digital, puesto que Internet estará presente en la TDT en un futuro próximo.

La interactividad rompe la tradicional comunicación vertical y unidireccional de la televisión convencional y la obliga a reinventarse de nuevo para adaptarse a los nuevos tiempos.

La interactividad supone una experiencia distinta a la hora de situarse ante la televisión, y permite abandonar la actitud pasiva tradicional por otra mucho más activa y participativa. El ciudadano interacciona con la pantalla, busca y obtiene información, ejerce su ciudadanía, por ejemplo, en 
servicios de impuestos, de búsqueda de trabajo, de información de tráfico, meteorológica, de bolsa, etc.

Además, por la facilidad de navegabilidad, cuatro botones de colores en los mandos a distancia, reduce la brecha tecnológica que se produce en la sociedad actual, bien por desconocimiento del uso de las nuevas tecnologías, bien por cuestiones económicas, bien por una actitud de hostilidad ante los nuevos medios.

\section{Claves para el éxito de la Televisión Digital Terrestre}

A modo de conclusión, habría que señalar algunas claves para analizar los distintos modelos de implantación de la Televisión Digital Terrestre y sus perspectivas de éxito:

\section{Primera: Necesidad de partir de modelos gratuitos}

La experiencia ha demostrado que el principal motor de penetración de la Televisión Digital Terrestre son los modelos libres y no las plataformas de pago.

Los gobiernos tienen que realizar esfuerzos en este sentido desde el convencimiento que una televisión digital gratuita, donde convivan operadores públicos y privados, es el modelo necesario para disminuir las diferencias económicas, sociales, culturales e incluso tecnológicas entre los ciudadanos, diríamos que esta opción es una necesidad social en el sentido que hemos defendido públicamente en más de una ocasión: 
En las sociedades contemporáneas, desde hace ya bastantes años, no es discutible que el sistema de enseñanza, o el sanitario, o la vivienda, o las pensiones, o la red de servicios destinados a las personas mayores... responden a necesidades sociales a las que las Administraciones han de hacer frente. Pero también se ha venido consolidando la idea de que el derecho a una información veraz plural e imparcial, el derecho a la cultura y a la educación, a un ocio creativo, es, también una necesidad social.

A este respecto, conviene recordar un fragmento procedente del Informe McBride de la UNESCO que fue publicado no hace un mes, ni un año, ni siquiera hace cinco años. Fue hecho público en 1980, hace casi un cuarto de siglo.

Dice así:

Si hay cientos de millones de mujeres y de hombres privados de los instrumentos indispensables de la comunicación, ¿qué sentido tiene hablar del derecho a informar o a ser informados? Es, pues, indispensable acopiar todos los medios posibles —educativos, culturales y sociales-, combinados con las diversas técnicas de comunicación y con los medios de comunicación social, para eliminar esta tara que empaña las perspectivas de todos los países del mundo.

En cualquier caso, el modelo actual de la Televisión Digital Terrestre, por el que se ha optado en España y en Europa, garantiza el acceso a las ventajas de la Televisión digital a todos los hogares, incluso a los más modestos, a los que hasta ahora estaban olvidados por el modelo digital de pago. 
Segunda opòrtunidad: La Televisión Digital Terrestre permite una gran variedad de programas (donde ahora hay un canal en analógico, caben cuatro en digital y con mayor calidad de imagen y sonido) que permitirán la convivencia entre contenidos generalistas y temáticos, que fomentarán las funciones informativas y educativas pero también sin duda las de entretenimiento (no olvidemos que consumir televisión es una de las preferencias de los ciudadanos en su tiempo libre). Multiplicidad de canales que permitirán explorar nuevas fórmulas de hacer televisión (laboratorio de contenidos I+D+I) que acerquen a nuevos públicos. Multiplicidad de canales que permitan mantener el equilibrio y una sana competencia entre lo público y lo privado.

Tercera oportunidad: Buscar la complementariedad de ámbitos geográficos. El modelo geográfico de la Televisión Digital Terrestre debe buscar la complementariedad entre lo nacional y lo local. Las televisiones de proximidad deben explorar las posibilidades que ofrecen los servicios interactivos municipales, las Televisiones de proximidad tienen una ventana de oportunidad siempre que sean capaces de aunar eficiencia en la gestión con un servicio diferenciado a los espectadores. Y sin olvidar que la interactividad puede ser la gran baza para que las televisiones locales permitan gestionar multitud de servicios municipales a través del televisor digital.

La cuarta oportunidad, por tanto, que nos permite la Televisión Digital Terrestre es apostar por la innovación y la interactividad y extender la Televisión Digital Terrestre a nuevas plataformas multimedia.

Hemos defendido y seguimos defendiendo que el desarrollo de la Televisión Digital Terrestre, de sus contenidos, de su accesibilidad, del desarrollo de su potencial interactivo ha de hacerse con visión de futuro, es decir pensando en generalizar el acceso a la Sociedad de la Información, a la Sociedad del Conocimiento y evitar que se profundice en la llamada "brecha digital". 


\section{Referencias bibliográficas}

AA VV (2006): “Análisis prospectivo acerca del futuro de la reforma audiovisual”, Tendencias 06

Medios de Comunicación. El año de la Televisión. Fundación Telefónica, 2006 pp.337- 351

Anuario de la televisión 2005/06, (2006), GECA

CAFFAREL, C. (2007): "Los medios de comunicación ante la España actual”, Hacia la radiotelevisión Española de los ciudadanos, Laberinto, Madrid, pp 83-100.

CAFFAREL, C. (2007): "El servicio público de televisión en España”, Journal of Spanish Cultural Studies, Routledge, Vol. 8, No 1, marzo,

FERNÁNDEZ BEAUMONT, J. (2006): “La refundación del sector audiovisual”, Tendencias 06 Medios de Comunicación. El año de la Televisión. Fundación Telefónica, 2006, pp 221- 242

LAMAS ALONSO, C. y PASCUAL ADRIÁN, J. (Coord) (2006): El libro verde de los canales temáticos. Una perspectiva multidisciplinar de la publicidad en los canales temáticos. Zenithmedia, Arce media, AIMC 
TELEVISIÓN DIGITAL. MARZO 2005. Grupo de Análisis y Prospectiva del Sector de las Telecomunicaciones (GAPTEL) Red.es. Ministerio de Industria, Turismo y Comercio.

www.impulsadtd.es 